\title{
Perspektiven für eine konzeptionelle Orientierung der Bildungsgeographie
}

\author{
T. Freytag ${ }^{1}$ and H. Jahnke ${ }^{2}$ \\ ${ }^{1}$ Albert-Ludwigs-Universität Freiburg, Institut für Umweltsozialwissenschaften und Geographie, \\ 79085 Freiburg, Germany \\ ${ }^{2}$ Europa-Universität Flensburg, Interdisziplinäres Institut für Umwelt-, Sozial- und Humanwissenschaften, \\ Abteilung Geographie, Auf dem Campus 1, 24943 Flensburg, Germany \\ Correspondence to: H. Jahnke (holger.jahnke@uni-flensburg.de)
}

Received: 16 April 2014 - Revised: 15 August 2014 - Accepted: 19 August 2014 - Published: 25 February 2015

Kurzfassung. Education has become central to the social and political debates in many countries. Under the influence of comparative international studies as well as national rankings of education institutions there is a growing awareness for social and regional inequalities of formal education infrastructure and processes. This article focuses on the geographical education research in Germany by reviewing existing research work on the one hand and opening perspectives for future studies in geography of education on the other. The main aim is to restructure the field of German Bildungsgeographie (geography of education) along key concepts and perspectives from human geography. After a brief introduction, the first part discusses the concept of Bildung (education) and its transformation. The following chapter sketches the major lines of research in geography of education in the German-speaking context. In the last and most extensive part six key concepts from human geography are pointed out as suitable reference points to situate existing and future research activities in the field of geography of education.

\section{Einleitung}

Das anhaltende Interesse an PISA-Ergebnissen und anderen Studien zum Vergleich der Leistungen von Schülerinnen und Schülern sowie die Umstrukturierung des Studiums an europäischen Hochschulen im Zuge des Bologna-Prozesses verdeutlichen beispielhaft die zentrale Stellung, die Bildung und Bildungssysteme in aktuellen Gesellschaften einnehmen. Es handelt sich dabei um eine Entwicklung von globaler Dimension, die häufig mit dem Begriff der Wissensgesellschaft assoziiert wird (Stehr, 1994). Nicht nur Bildungseinrichtungen, Bildungsinhalte und Curricula werden von der aktuellen Dynamik erfasst, sondern auch die an der Vermittlung von Bildung und Wissen beteiligten Akteure nehmen an diesem umfassenden und vielschichtigen Transformationsprozess teil (Autorengruppe Bildungsberichterstattung, 2012). Innerhalb Europas lassen sich eine fortschreitende Expansion des Bildungswesens und eine Pluralisierung sowie Diversifizierung der Bildungsangebote beobachten (OECD, 2013).
Dabei ist der institutionalisierte Bildungserwerb zunehmend durch Tendenzen zur Privatisierung, Ökonomisierung und Internationalisierung geprägt.

Bei der wissenschaftlichen Untersuchung bildungsbezogener Phänomene wird deren räumliche Dimension häufig vernachlässigt. Umgekehrt werden die aktuellen Bildungsdebatten in der Geographie nur wenig reflektiert und die gesellschaftliche Bedeutung bildungsgeographischer Forschung offensichtlich unterschätzt. Diesen Eindruck gewinnt man zumindest bei der kursorischen Sichtung deutschsprachiger geographischer Fachzeitschriften und Lehrbücher. Zwar besteht in Deutschland eine Forschungstradition der Bildungsgeographie, sie erscheint jedoch noch nicht in ausreichendem Maße in die interdisziplinäre Bildungsforschung eingebunden und nimmt auch innerhalb der deutschsprachigen Humangeographie - ungeachtet der hohen gesellschaftlichen und politischen Relevanz bildungsbezogener Themen meist nur eine Randstellung ein. So erklärt es sich, dass z.B. im Bereich der Neuen Kulturgeographie theoretische und 
empirische Untersuchungen zu Bildung und Wissen über längere Zeit weitgehend unberücksichtigt geblieben sind. Während im angelsächsischen Raum in der geography of education and learning jüngst eine zunehmende und zum Teil kritische Auseinandersetzung mit Restrukturierungen im angelsächsischen Bildungswesen stattfindet (Holloway et al., 2010), werden die grundlegenden Transformationsprozesse in den deutschen Bildungslandschaften bisher kaum in der geographischen Forschung und Lehre reflektiert (Jahnke, 2014). Dennoch gibt es im deutschsprachigen Raum bereits seit Mitte der 1960er Jahre eine eigenständige bildungsgeographische Forschungstradition (Meusburger, 1998).

Der vorliegende Beitrag ordnet die vorhandenen Arbeiten vor dem Hintergrund bestehender Basiskonzepte in der Humangeographie und benennt abschließend Desiderata für eine künftige bildungsgeographische Forschung. Das zentrale Ziel dieses Beitrags besteht darin, das Forschungsfeld der Bildungsgeographie entlang grundlegender humangeographischer Konzepte und Perspektiven unter besonderer Berücksichtigung der deutschsprachigen Debatte neu zu strukturieren und den Weg zu einer kritischen Bildungsgeographie zu öffnen. Dazu wird zunächst der Bildungsbegriff innerhalb sozial- und wirtschaftswissenschaftlicher Zusammenhänge eingeordnet. Es folgt eine knappe Darstellung der Entwicklungslinien des Forschungsfeldes der Bildungsgeographie.

\section{Bildung, Wissen und Kompetenz}

Während der zurückliegenden Jahrzehnte bildungsgeographischer Forschung unterlag der Bildungsbegriff einem starken Wandel; gleichzeitig haben die Begriffe Wissen und Kompetenz Eingang in die interdisziplinäre Bildungsforschung gefunden. Eine zeitgemäße Bildungsgeographie sollte folglich nicht nur den aktuellen humangeographischen Strömungen Rechnung tragen, sondern auch eine Verortung innerhalb der breiteren Bildungsdebatte ermöglichen. Vor dem Hintergrund einer zunehmenden Unschärfe von Begrifflichkeiten im Themenfeld Bildung und der wachsenden Komplexität von Bildungsdiskursen, -institutionen und -verhalten wird hier zunächst der Begriff der Bildung mit Blick auf das bildungsgeographische Interesse skizziert und in bestehende semantische Begriffsfelder eingeordnet.

Bildung wurde lange Zeit in der Tradition von Humanismus und Aufklärung als die zweckfreie Entfaltung von Individuen in deren Auseinandersetzung mit sich selbst und mit der sozialen und materiellen Umwelt erachtet. Der Bildungstheoretiker Wilhelm von Humboldt verstand Bildung als Prozess der Wechselwirkung zwischen dem Ich und der Welt, gewissermaßen als eine dialogische Beziehung, welche in der Sprache ihren Rahmen findet. Diese allgemeine und zweckfreie Bildung sollte durch ein dreistufiges Bildungswesen - von der Elementarbildung zur schulischen und universitären Bildung - eine geeignete institutionelle Verankerung erhalten. Im ausgehenden 18. und beginnenden 19. Jahrhun- dert wurde das aus Primar-, Sekundar- und Hochschulbildung bestehende Grundgerüst des Bildungssystems sowohl für Deutschland als auch im Hinblick auf die Entwicklung der internationalen Bildungslandschaft gelegt (Dörpinghaus und Uphoff, 2012:76-85).

Mit der Bildungsexpansion der 1960er und 1970er Jahre rückte Bildung zusehends in das Zentrum gesellschaftspolitischer Debatten (Picht, 1964). Getragen von der Leitidee der Gerechtigkeit von Bildungschancen wurde das komplexe Phänomen Bildung vermehrt mittels statistischer Indikatoren (z.B. Übertritts-, Absolventen- und Feminisierungsquoten) formalisiert und bestehende Bildungsungleichheiten wurden sichtbar gemacht. Diese waren auch Grundlage der frühen Bildungsgeographie als einer quantitativ ausgerichteten sozialgeographischen Forschungsrichtung, die u.a. raumbezogene Ungleichheiten betonte (Geipel, 1969; Aurin, 1970).

Seit den 1980er Jahren ist die Betrachtung der (sozio-)ökonomischen Dimension von Bildung als „Humankapital“ im Sinne von verwertbaren Qualifikationen und Wissen als ökonomischem Gut stärker in das Zentrum des wissenschaftlichen und gesellschaftlichen Interesses gerückt. Dabei hat der Bildungsbegriff selbst an Konturschärfe eingebüßt und wurde teilweise durch die Termini Wissen und Kompetenz überlagert und semantisch verschoben.

Der Begriff des Wissens steht zunächst in engem Zusammenhang mit Bildungsprozessen und daher auch mit der bildungsgeographischen Forschung. Vor allem Meusburger hat entscheidende Grundlagen für eine umfassende und interdisziplinär verankerte geography of knowledge, science and education gelegt und damit die Geographien des Wissens, der Wissenschaft und der Bildung zueinander in Beziehung gesetzt. Dabei betont er die Mehrdimensionalität des Wissensbegriffs, die er aus anderen Disziplinen wie z.B. den science studies oder den Kultur- und Religionswissenschaften ableitet. Einerseits existieren sehr unterschiedliche Formen des Wissens, die in verschiedene kulturelle oder soziale Kontexte oder Systeme eingebettet sind, andererseits können diese unterschiedlichen Wissensformen als ökonomische, soziale, kulturelle wie auch politische Ressourcen fungieren (Meusburger, 2010).

Als Folge des sogenannten PISA-Schocks wurde die deutsche Bildungsdebatte seit den 1990er Jahren von der Frage nach Kompetenzen und damit nach erlernbaren Fähigkeiten und Fertigkeiten bestimmt (Weinert, 2001:27f.). Bildungsziele werden seitdem weniger über Fachinhalte definiert als über Kompetenzanforderungen, die in Form national gültiger Bildungsstandards festlegen, welche Fähigkeiten und Fertigkeiten die Schülerinnen und Schüler einer bestimmten Jahrgangsstufe mindestens erworben haben sollten. Durch diese Form der Kompetenzmessung von Lernenden wird gleichzeitig auch die Leistungsfähigkeit ganzer Bildungssysteme evaluiert. Denn mit den nun standardisierten Variablen liegen vergleichbare Output-Messungen vor, die als Instrumente des Bildungsmonitoring eingesetzt werden können (Klieme et al., 2007:10), gleichzeitig aber auch neue Potentiale für eine 


\section{Informelles Lernen}

in alltäglichen Zusammenhängen der Lebenswelt, nicht didaktisch organisiert,

nicht immer als Lernfortschritt wahrgenommen

\section{Non-formale Bildung}

in sonstigen Bildungseinrichtungen erworben

(z.B. Jugendarbeit, Sportvereine, Musikschulen);

ohne anerkannte Abschlüsse

\section{Formale Bildung}

in Bildungs- und Ausbildungseinrichtungen

für die allgemeine und berufliche Bildung

erworben (z.B. Schulen, Hochschulen);

mit anerkannten Abschlüssen

Abb. 1. Bildungsbegriffe und Bildungsräume. Quelle: Autorengruppe Bildungsberichterstattung (2012), VIIIf.; eigene Darstellung.

quantitative und zudem räumlich differenzierte Bildungsforschung eröffnen. Aus Sicht eines humanistischen Bildungsideals verbindet sich mit der Kompetenzorientierung jedoch die Einengung auf einen funktionalistischen Bildungsbegriff, der die Entwicklung arbeitsmarktrelevanter Fertigkeiten gegenüber der freien Persönlichkeitsentwicklung priorisiert.

Somit liegt beiden Debatten - sowohl der Kompetenzdebatte aus den Bildungswissenschaften als auch der Wissensdebatte aus Ökonomie, Soziologie und teilweise der Geographie - zumindest in ihrem breiten gesellschaftspolitischen Diskurs ein dominierender Funktionalismus zugrunde, der nach dem gesellschaftlichen oder ökonomischen Zweck von Wissen und Kompetenzen fragt. Auch Nico Stehr, Soziologe und einer der führenden Theoretiker der Wissensgesellschaft, gelangt - aus handlungstheoretischer Perspektive - zu einer funktionalistischen Minimaldefinition von Wissen als ,,capacity for (social) action" (Stehr, 1996), die in dieser kurzen Form der oben angeführten Definition von Kompetenz sehr nahe kommt. Mit der inhaltlichen Verschiebung der aktuellen Bildungsdebatte in Richtung Wissen und Kompetenz verbindet sich somit die Dominanz einer funktionalistischen Perspektive auf Bildungsprozesse und zugleich die Distanzierung von einem zweckfreien humanistischen Bildungsideal.

Neben den geschilderten theoretischen Verschiebungen der akademischen und gesellschaftlichen Bildungsdiskurse zeichnen sich in den letzten Jahren auch im Bereich der bildungspolitischen Praxis deutliche Veränderungen des Bildungsverständnisses auf institutioneller Ebene ab. Diese äuBern sich vor allem in einer Neuverortung von formalen, nonformalen und informellen Bildungspraktiken, deren institutionelle Grenzen zunehmend verschwimmen (Abbildung 1). Der Begriff der formalen Bildung bezieht sich auf Bildungs- prozesse, die in Bildungseinrichtungen stattfinden und durch formale Bildungsabschlüsse zertifiziert werden. Demgegenüber findet non-formale Bildung meist auch innerhalb eines institutionellen Rahmens statt (z.B. Musikschule, Volkshochschule, Sportverein, Kultur- oder Jugendzentrum); die erworbenen Kenntnisse, Fertigkeiten oder Qualifikationen werden jedoch nicht als formale Bildungsabschlüsse anerkannt und gehen bisher nicht in die Bildungsstatistik ein. Informelles Lernen vollzieht sich hingegen primär außerhalb von Bildungsinstitutionen, beispielsweise in der Familie, beim Spielen in der Natur, auf dem Weg zur Schule oder beim Surfen im Internet. Mit Blick auf die Schulentwicklung der letzten Jahre, insbesondere im Bereich des offenen Ganztags, ist ein Trend zur stärkeren Verknüpfung der Bildungsarbeit unterschiedlicher Institutionen zu beobachten (Autorengruppe Bildungsberichterstattung, 2012).

Die regionale Bildungsforschung in Deutschland - und auch die klassische Bildungsgeographie - hat ihren Schwerpunkt lange Zeit auf die Untersuchung formaler und damit statistisch dokumentierter Bildungsprozesse gelegt (Weishaupt, 2009a). Vor dem Hintergrund eines offenen Bildungsbegriffs, der non-formale und informelle Bildungsprozesse stärker berücksichtigt, können auch in der bildungsgeographischen Forschung formale Bildungsprozesse nicht länger isoliert betrachtet werden. Nicht nur der nationale Bildungsbericht, sondern auch die aktuellen Arbeiten der angelsächsischen geography of education sowie des Arbeitskreises Bildungsgeographie der Deutschen Gesellschaft für Geographie tragen eben jener Entwicklung Rechnung, lassen aber gleichzeitig einen erheblichen Forschungsbedarf im Bereich der non-formalen und informellen Bildung in Deutschland erkennen.

\section{Entwicklungslinien der Bildungsgeographie}

Im zeitlichen Kontext der Münchner Schule der Sozialgeographie, deren Vertreter u.a. die Daseinsgrundfunktion ,sich bilden" in ihrer räumlichen Dimension betrachteten, entwickelte sich im deutschsprachigen Raum um Robert Geipel seit Mitte der 1960er Jahre eine eigenständige bildungsgeographische Forschungstradition (Geipel, 1966, 1968, 1976; Meusburger, 1976, 1998; Wagner, 1993). Unter dem Eindruck der fortschreitenden Bildungsexpansion, die auch mit der Gründung und dem Ausbau zahlreicher Schul- und Hochschulstandorte verbunden war, wurden bildungsbezogene Strukturen und Prozesse als gesellschaftlich relevant erkannt und rückten zunehmend in den Fokus des wissenschaftlichen Interesses. Eine intensive Begleitforschung machte es sich zur Aufgabe, den Prozess einer sukzessiven räumlichen Bildungsexpansion zu dokumentieren und zu analysieren. Dabei wurden seitens der Wissenschaft beratende Funktionen eingenommen und im Rahmen von Forschungsarbeiten substantielle Beiträge für die Bildungsplanung geleistet (u.a. Geipel, 1969, 1971; Mayr, 1970, 1979; Bahrenberg, 1974; Meusbur- 


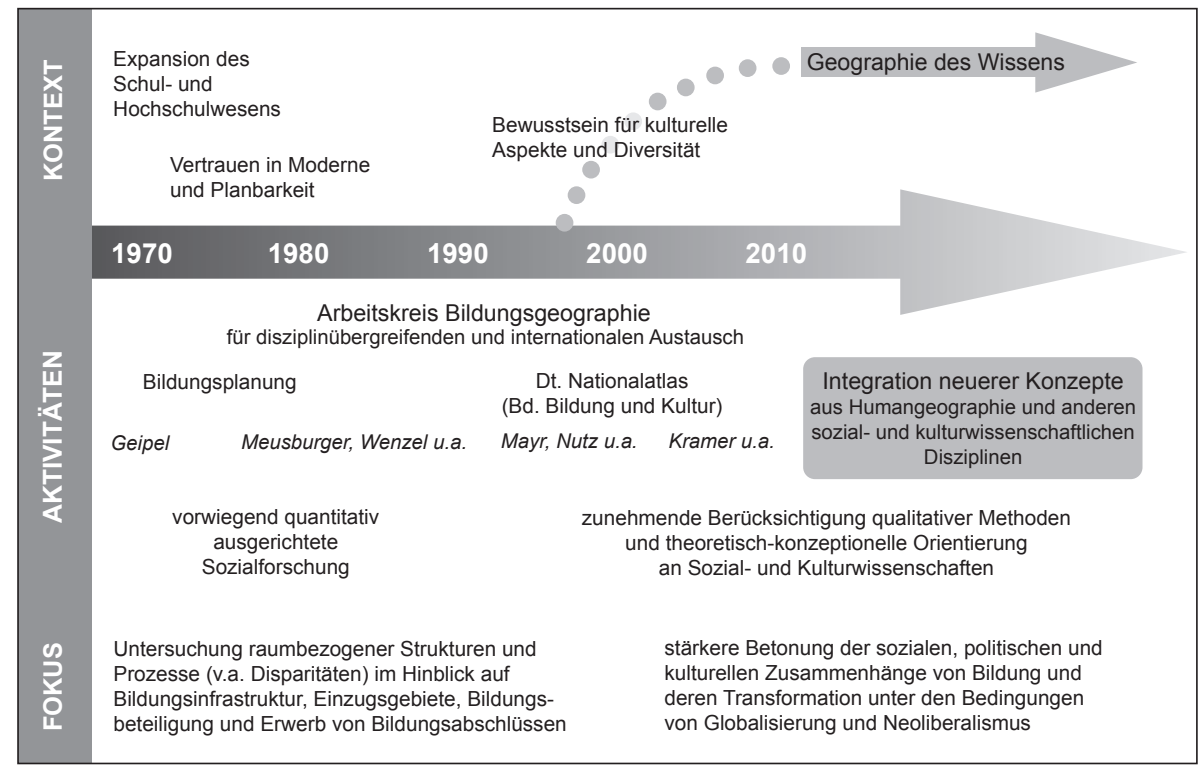

Abb. 2. Entwicklungslinien der Bildungsgeographie im deutschsprachigen Raum. Quelle: eigene Darstellung.

ger, 1974, 1980; Monheim, 1977, 1983; Giese, 1982). Vor diesem Hintergrund konstituierte sich schließlich eine interdisziplinäre Forschungsgruppe, deren Zusammenschluss zum Arbeitskreis Bildungsgeographie der Deutschen Gesellschaft für Geographie 1983 erfolgte (Abbildung 2).

Im Rahmen des AK Bildungsgeographie wurden daraufhin weitere empirisch geprägte und teilweise mit einem raumplanerischen Anwendungsbezug versehene Studien durchgeführt. Diese beschäftigten sich z.B. mit der Bildungsversorgung (Steingrube, 1984; Kramer, 1993), den Einzugsgebieten von Hochschulen (Nutz, 1991) sowie den räumlichen Disparitäten des Ausbildungsniveaus der Bevölkerung (Kramer, 1998). Ein besonderes Augenmerk galt der beruflichen Ausbildung und der Eingliederung in den Arbeitsmarkt (Wenzel et al., 1990; Rolfes, 1996) sowie den regionalen Effekten von Hochschulen und Hochschulneugründungen (Giese, 1987; Schamp, 1989). Weiterhin wurden die Feminisierung des Lehrerberufs (Schmude, 1988) und die räumliche Mobilität von Hochqualifizierten (Meusburger, 1986; Weick, 1995) untersucht. Dabei richtete sich das Forschungsinteresse nicht allein auf den deutschsprachigen Raum, sondern suchte gelegentlich auch eine internationale Perspektive. Dies kommt z.B. in Arbeiten zum Hochschulwesen in Japan (Flüchter, 1990), zur historischen Alphabetisierung in England (Hoyler, 1995, 1998) sowie zu Bildung und Ethnizität in den USA (Frantz, 1994; Freytag, 2003a, b; Gamerith, 2005a, b) und Hochschulabsolventinnen und absolventen in Italien (Jahnke, 2005) zum Ausdruck.

Mit dieser Themenvielfalt etablierte sich die Bildungsgeographie zusehends als eigenständige sozialgeographische Teildisziplin, die Bildungswesen und Bildungserwerb als Schlüssel zum Verständnis gesellschaftlicher Strukturen und
Prozesse versteht. Methodisch dominierten zunächst empirische Untersuchungen, in deren Rahmen vorhandene Sekundärstatistiken analysiert und ergänzend auch eigene quantitative Erhebungen durchgeführt wurden. Insbesondere in Deutschland erwies sich die Verfügbarkeit und Qualität von Sekundärdaten der amtlichen Statistik aufgrund der Kulturhoheit der Länder nicht selten als unbefriedigend und teilweise sogar als eine faktische Forschungsbarriere. Einen anschaulichen Überblick über das Themenspektrum einer empirisch ausgerichteten bildungsgeographischen Forschung seit den 1980er Jahren vermitteln Meusburger und Schmude (1990) sowie vor allem der auf Initiative von Mayr und Nutz herausgegebene Nationalatlas-Band „Bildung und Kultur“ (Institut für Länderkunde et al., 2002), auf dessen Grundlage später eine zusammenfassende Aktualisierung und Überarbeitung einzelner Beiträge vorgenommen wurde (Kramer und Nutz, 2010).

Impulse für eine Öffnung gegenüber theoretischen Konzepten aus den Sozial-, Kultur- und Geisteswissenschaften haben die deutschsprachige Tradition der Bildungsgeographie seit Ende der 1990er Jahre geprägt. Das Interesse richtet sich nun zunehmend auf disziplinübergreifende Theorien und Konzepte und den vermehrten Einsatz qualitativer Verfahren. In diesen Arbeiten liegt die Betonung auf der kulturellen Bedingtheit von Bildungserwerb und Bildungsinstitutionen sowie auf bildungsbezogenen Entscheidungen und Praktiken (z.B. Freytag, 2003a; Jahnke, 2005). Parallel zu dieser Veränderung ist seit Ende der 1990er Jahre auch zu beobachten, dass sich aus der Bildungsgeographie eine eigenständige Geographie des Wissens bzw. geography of knowledge mit starken Bezügen zur geography of science entwickelt (Meusburger, 2000, 2005; Jöns, 2003; Jahnke, 2004; 
Meusburger et al., 2008). Da der Schwerpunkt dieser Forschungsrichtung jedoch weniger im Bereich der allgemeinen Bildung angesiedelt ist und nur wenige Anknüpfungspunkte zur klassischen Bildungsgeographie bestehen, soll die geography of knowledge im vorliegenden Beitrag nicht vertiefend betrachtet werden.

Unter dem Eindruck internationaler Vergleichsstudien rückt die Bildungsforschung im beginnenden 21. Jahrhundert wieder stärker in das Interesse der Öffentlichkeit. Dies begünstigt auch die Entstehung neuer bildungsgeographischer Arbeiten, die sich teilweise auf Geographische Informationssysteme (GIS) stützen, um raumbezogene Strukturen und Prozesse der Bildungsversorgung und des Ausbildungsniveaus der Bevölkerung vor dem Hintergrund des demographischen Wandels in Deutschland zu untersuchen (z.B. Terpoorten, 2005; Frank, 2011). Parallel werden in der bildungsgeographischen Forschung weiterhin qualitative Methoden eingesetzt, so dass für den deutschsprachigen Raum eine starke empirische Fundierung und eine Kombination bzw. ein Nebeneinander von quantitativen und qualitativen Verfahren charakteristisch bleiben (Pfaffenbach, 2006; Güles et al., 2010; Müller, 2010; Schamp und Zajontz, 2010).

Im englischsprachigen Raum ist derzeit das Aufleben einer nahezu ausschließlich qualitativ ausgerichteten geography of education zu beobachten, die jedoch ausführlichere Bezüge zur deutschsprachigen Forschung bislang vermissen lässt (Butler und Hamnett, 2007; Hanson Thiem, 2008; Barker et al., 2010; Cook und Hemming, 2010; Holloway et al., 2010; Hemming, 2011). Unter dem Namen geography of education and learning entwickelt sich zudem eine Forschungsrichtung, die sowohl eine Brücke zur Kindheits- und Jugendforschung (children's geographies) schlägt (Holloway et al., 2010; Holloway und Jöns, 2012) als auch den Blick verstärkt auf Orte und Einrichtungen der non-formalen und informellen Bildung richtet (Kraftl, 2013).

Eine theoretisch-konzeptionelle Ausrichtung der Bildungsgeographie als dezidiert geographische Perspektive innerhalb der deutschsprachigen interdisziplinären Bildungsforschung steht noch aus. Im Folgenden werden erste Überlegungen zu einer solchen Neuordnung des bildungsgeographischen Forschungsfelds entwickelt, die künftigen Forschungsarbeiten als Anregung und Orientierung dienen können.

\section{Geographische Konzepte für die Bildungsforschung}

In weiten Teilen der Humangeographie wurde seit den 1980er Jahren der Trendwechsel von einer klassischen Raumwissenschaft zu einer dynamischen Kultur- und Sozialwissenschaft vollzogen. Im Zuge dieses Wandels hat sich das Forschungsinteresse stärker auf theoretisch-konzeptionelle Fragestellungen konzentriert. Dabei richtete sich ein besonderes Augenmerk auf die Auseinandersetzung mit dem durch

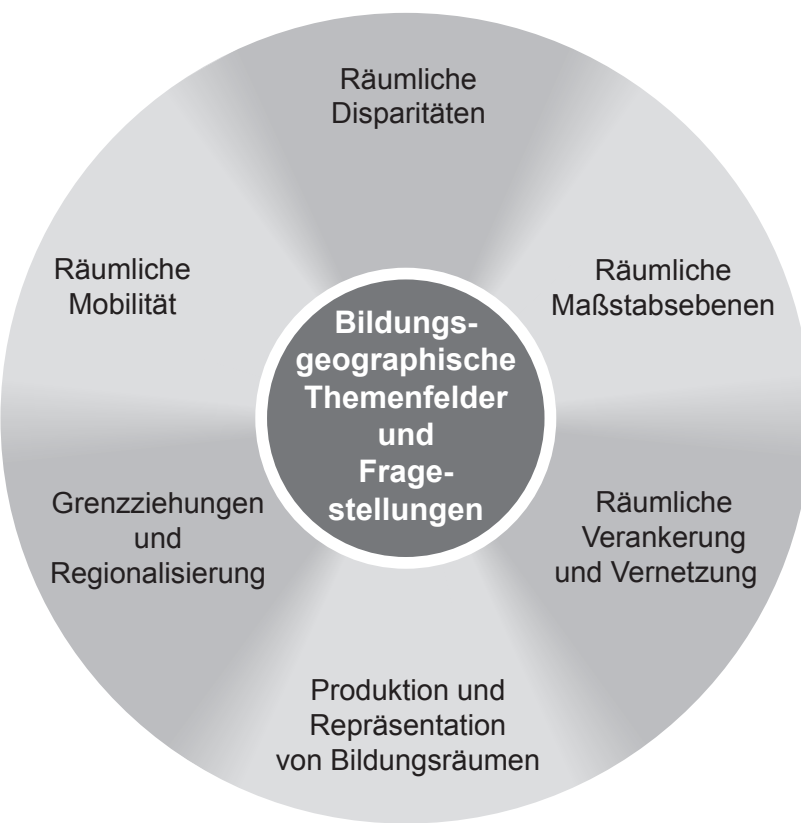

Abb. 3. Ausgewählte geographische Konzepte für die bildungsgeographische Forschung. Quelle: eigene Darstellung.

komplexe Wechselwirkungen geprägten Verhältnis zwischen Raum, Gesellschaft und Handlung. Unter dem Einfluss der Neuen Kulturgeographie wurden ein interdisziplinärer Austausch mit den Sozial- und Kulturwissenschaften sowie eine intensivere Beschäftigung mit qualitativen Forschungsmethoden angeregt. Vor diesem Hintergrund soll nun dargelegt werden, wie existierende geographische Forschungskonzepte als strukturierende Leitlinien für eine raumbezogene Bildungsforschung herangezogen werden können. Im Einzelnen werden dabei sechs ausgewählte geographische Konzepte etwas eingehender betrachtet (Abbildung 3).

Die Auswahl dieser sechs geographischen Konzepte orientiert sich an laufenden Diskussionen über Grundkategorien des geographischen Denkens einerseits (z.B. Clifford et al., 2009) und der Betrachtung der bisherigen bildungsgeographischen Forschungspraxis andererseits. Die hier verfolgte pragmatische Auswahl erhebt keinen Anspruch auf Vollständigkeit, sondern dient vielmehr dazu eine Debatte anzustoßen. Es wird sich jedoch zeigen, dass die genannten Konzepte geeignet sind, verschiedene Themenfelder der Bildungsforschung perspektivisch zu strukturieren und als analytischer Rahmen für künftige Untersuchungen zu fungieren. In den folgenden Teilkapiteln werden die einzelnen geographischen Konzepte jeweils kurz vorgestellt und anschließend in ihrer Bedeutung für bildungsgeographische Forschungsarbeiten erörtert. Es wird deutlich, dass bildungsbezogene Aspekte jeweils im Hinblick auf Institutionen (z.B. Schulen und Hochschulen) und Akteure (z.B. Lernende und Lehrende) analysiert werden können. Weiterhin wird ausgeführt, dass sich auch Bildungsdiskurse in ihrer raumbezogenen 
Artikulation als Forschungsgegenstand untersuchen lassen. Bei der getrennten Behandlung der verschiedenen geographischen Konzepte handelt es sich lediglich um eine analytische Trennung, die in dem Bewusstsein vorgenommen wurde, dass die anhand der einzelnen Konzepte untersuchten bildungsbezogenen Phänomene selbstverständlich auch ineinandergreifen und sich wechselseitig beeinflussen können.

\subsection{Räumliche Disparitäten}

Wenn Personen, Objekte, Institutionen oder Merkmale nicht gleichmäßig im Raum verteilt auftreten, handelt es sich um räumliche Disparitäten. Eine Aufgabe der geographischen Forschung kann darin bestehen, diese Ungleichheiten als räumliche Muster zu erfassen sowie deren Entstehungsund Wirkungszusammenhänge zu analysieren (Ward, 2009). Weiterhin kann der Frage nachgegangen werden, wie vorhandene räumliche Ungleichheiten von einem normativen Standpunkt aus bewertet werden und inwieweit seitens der Wissenschaft, Politik und Gesellschaft versucht werden sollte, räumliche Disparitäten abzubauen und auf diese Weise einen Ausgleich herbeizuführen.

Ein zentrales Thema der Bildungswissenschaften, das in der Öffentlichkeit intensiv und kontrovers diskutiert wird, sind die bestehenden bildungsbezogenen Ungleichheiten, die anhand verschiedener Indikatoren dokumentiert und sichtbar gemacht wurden und werden (Autorengruppe Bildungsberichterstattung, 2012; OECD, 2013). Üblicherweise werden diese Ungleichheiten primär als ein allgemeines gesellschaftliches Problem wahrgenommen, das weitgehend losgelöst von räumlichen Zusammenhängen existiert. Wie jedoch der Band „Bildung und Kultur“ im Nationalatlas Bundesrepublik Deutschland (Institut für Länderkunde et al., 2002) anhand zahlreicher Beispiele verdeutlicht, ist die deutsche Bildungslandschaft durch vielfältige räumliche Disparitäten geprägt. Dies betrifft z.B. die Anordnung von Bildungseinrichtungen im Raum und ebenso das Ausbildungsniveau der Bevölkerung.

Im Hinblick auf die Bildungseinrichtungen ist offensichtlich, dass diese ungleich im Raum verteilt sind. Es gibt Standorte, die über eine ausgezeichnete Versorgung mit Schulen und Hochschulen verfügen, während die vorhandene Bildungsinfrastruktur an anderen Standorten deutliche Defizite aufweist. So konzentrieren sich vor allem höhere Bildungseinrichtungen in den oberen Stufen der Siedlungshierarchie, während ländliche und periphere Räume oft Versorgungsdefizite beklagen (Kramer, 1993). Eine geographische Betrachtungsweise verdeutlicht, dass das gesellschaftliche Leitziel der flächendeckend gleichwertigen Lebensverhältnisse eine illusionistische Vorstellung ist, da dem Raum eine Heterogenität im Hinblick auf die infrastrukturelle Ausstattung und die damit verbundenen Lebensbedingungen quasi inhärent ist.
Aufgabe der Raumplanung ist es, vor diesem Hintergrund eine sinnvolle und ausgewogene Bildungsinfrastruktur herzustellen, die der Bevölkerung an verschiedenen Wohnstandorten den Zugang zu möglichst guten Ausbildungschancen eröffnet. Unter dieser Prämisse wurde seit den 1970er Jahren die Planung von Schulen und Hochschulen in Deutschland betrieben (Geipel, 1969; Monheim, 1983). Auch in Gebieten mit rückläufigen Bevölkerungszahlen spielen Fragen der angemessenen Versorgung der dort lebenden Bildungsteilnehmerinnen und -teilnehmer eine wichtige Rolle, wenn es z.B. um einen gezielten Rückbau bzw. eine Schließung der Bildungsinfrastruktur geht (Weishaupt, 2009b; Müller, 2010; Frank, 2011).

Hinsichtlich der Bildungsbeteiligung und des Bildungserfolgs lassen sich ebenfalls deutliche bildungsbezogene Unterschiede in Form räumlicher Disparitäten beobachten. Zum Teil können diese in Wechselwirkung mit der Anordnung der Bildungsinfrastruktur und der räumlichen Struktur des Arbeitsmarktes verstanden werden. Dies erklärt z.B. die häufig zu verzeichnende Konzentration von Hochqualifizierten in Großstädten und Agglomerationsräumen (Meusburger, 1980, 2000).

Einen geographischen Analyseansatz für räumliche Disparitäten bietet die spatial analysis (Johnston, 2009). Orientiert an einem quantitativ empirisch ausgerichteten Forschungsparadigma leistet diese Form der Raumanalyse eine systematische Interpretation, die unmittelbar aus den zur Verfügung stehenden Daten abgeleitet wird. Auf diese Weise können räumliche Muster insbesondere in puncto Konzentration und Dekonzentration bzw. Homogenität und Heterogenität identifiziert und typisiert werden (Institut für Länderkunde et al., 2002). Die Grenzen dieses Ansatzes liegen zunächst in der eingeschränkten Verfügbarkeit valider Bildungsdaten. Ein weiteres Problem besteht darin, dass bildungsrelevante gesellschaftliche und kulturelle Rahmenbedingungen, die sich nicht auf der Grundlage von Daten einbeziehen lassen, bei der spatial analysis unberücksichtigt bleiben. Es lassen sich also raumbezogene Zusammenhänge aufzeigen und Regelmäßigkeiten feststellen, die aus den aggregierten Daten abgeleiteten Ursachen und Erklärungen bleiben jedoch meist unbefriedigend.

Um den Blick für bildungsbezogene Disparitäten als Ausdruck komplexer sozialer Phänomene und menschlicher Entscheidungsprozesse zu öffnen und Aspekte wie z.B. Macht und Politik zu berücksichtigen, sollten breiter gefasste und stärker qualitativ ausgerichtete humangeographische Forschungsansätze herangezogen werden. Es gilt dabei, bildungsbezogene Beobachtungen auch mit Blick auf andere konzeptionelle Zugänge zu kontextualisieren, d.h. mit sozioökonomischen und kulturellen Verhältnissen, Siedlungsstruktur und historischen Entwicklungen - im Sinne einer Pfadabhängigkeit - in Verbindung zu bringen. 


\subsection{Räumliche Mobilität}

Ein zweiter konzeptioneller Fokus richtet sich auf die bildungsbezogene räumliche Mobilität. Unter dem Begriff der räumlichen Mobilität wird traditionell zwischen Migration (d.h. Verlagerung des Wohnsitzes) und Alltagsmobilität bzw. Zirkulation unterschieden. Diese theoretische Trennung hat sich jedoch in Bezug auf die Beschreibung räumlicher Mobilität als immer weniger zutreffend erwiesen, so dass sich die aktuelle Migrationsforschung stärker auf hybride Konzepte wie Zirkulation oder Multilokalität stützt (Wehrhahn und Sandner Le Gall, 2011:91-94). Auf diese Weise sollen aktuelle weiträumige Pendel- und Aktionsraumverflechtungen konzeptionell gefasst und für die empirische Forschung greifbar gemacht werden. In der deutschen Bildungsgeographie wurden unterschiedliche Formen der räumlichen Mobilität bereits in der Vergangenheit bearbeitet (Weick, 1995; Rolfes, 1996), wobei vereinzelt auch Impulse für die theoretisch-konzeptionelle Entwicklung der Mobilitäts- und Migrationsforschung gesetzt wurden (Jöns, 2003).

Der grundsätzliche Zusammenhang zwischen Bildung und räumlicher Mobilität gilt schon lange als erwiesen (Meusburger, 1998:377-389). Denn einerseits können Bildungsaspirationen oder -angebote die Mobilitätsentscheidungen von Individuen oder Gruppen beeinflussen, andererseits steigert ein erhöhtes Ausbildungsniveau die Mobilitätswahrscheinlichkeit. Die Untersuchung von bildungsbezogener räumlicher Mobilität hat ihren Ursprung in der Bildungsplanung der 1960er Jahre, als es unter dem Primat der Gleichwertigkeit der Lebensverhältnisse und im Kontext der Schließung von Kleinschulen im ländlichen Raum darum ging, Regeln für die maximal zumutbare Pendeldistanz sogenannter „Fahrschüler“ festzulegen. Dabei konnte gezeigt werden, dass in Abhängigkeit von der spezifischen (bildungs-)geographischen Situation, also der räumlichen Distanz zur nächstgelegenen oder angestrebten Bildungsinstitution, schon der Besuch einer Grundschule, einer weiterführenden Schule oder einer Universität regelmäBiges Pendeln oder eine Wohnsitzverlagerung erforderlich macht. Daher führt räumliche Mobilität zur Verstärkung einer bereits vorhandenen Bildungskonzentration in den Zentren und damit auch zum Absinken des Qualifikationsniveaus in bildungsfernen Regionen. Dies trifft insbesondere auf das Migrationsverhalten von Studierenden (Nutz, 1991) sowie Hochschulabsolventinnen und -absolventen zu, die zum Verbleib an ihren Studienorten oder in den wirtschaftsstärkeren Zentren neigen (Rolfes, 1996; Jahnke, 2005).

Was im Kontext der interdisziplinären Migrationsforschung unter den Begriffen brain drain, brain gain, brain exchange oder skilled migration diskutiert wird, lässt sich aus bildungsgeographischer Perspektive auch als räumliche Mobilität inkorporierter Bildung verstehen. In der Frühphase der brain drain-Forschung lag der Fokus auf der Abwanderung von akademisch Hochqualifizierten aus den sogenannten „Entwicklungsländern“ nach Großbritannien und in die
USA (Meusburger, 1998:383f.), während sie sich seit den 1990er Jahren stärker auf die Migration aus den osteuropäischen Ländern (Rhode, 1993) nach Westeuropa oder Prozesse des brain drain innerhalb Deutschlands (Schultz, 2009) konzentrierte.

Die Forschung zur internationalen Migration von Hochqualifizierten hat jedoch auch gezeigt, dass mit dem brain drain aus einem Herkunftsland nicht automatisch ein brain gain im Zielland der Migration einhergeht. Wenn räumliche Mobilität mit einem Wechsel zwischen unterschiedlichen sozialen, kulturellen und sprachlichen Systemen verbunden ist, führt dies häufig zu einer Abwertung formaler Bildungsabschlüsse im Zielland. Dieses Phänomen wird in der Migrationsforschung als brain waste bezeichnet (Rhode, 1993; Fassmann et al., 1995) und verweist auf die nationalstaatliche Verankerung von Bildungsabschlüssen (vgl. Abschnitt 4.5).

Die Migrationsforschung hat sich in den letzten Jahrzehnten von der Vorstellung von räumlicher Mobilität als einer permanenten $\mathrm{Ab}$ - oder Zuwanderung gelöst und befasst sich nun stärker mit hybriden Konzepten wie skilled transients (Findlay, 1988), brain circulation (Friedrich und Schultz, 2008), Transnationalismus (Pries, 2003) und Multilokalität (Weichhart, 2009). Gerade unter jungen Akademikerinnen und Akademikern, deren Karriereverläufe strukturell mit Arbeitsortswechseln verbunden sind, unter Studierenden, die mehrere Hochschulen besuchen, oder im Fall von Wissenschaftlerinnen und Wissenschaftlern, die in Einzelfällen sogar Mitglieder mehrerer Universitäten oder Forschungseinrichtungen sein können, lassen sich derartige intermediäre Formen der interregionalen und internationalen Mobilität vermehrt beobachten. Auch die Auswirkungen bildungsbezogener Mobilität - z.B. mit Unterstützung durch internationale Mobilitätsprogramme für Schülerinnen und Schüler oder Studierende - wecken das Interesse der bildungsgeographischen Forschung (Weichbrodt, 2014).

\subsection{Räumliche Maßstabsebenen}

Bei der analytischen Betrachtung von Phänomenen und Themen auf unterschiedlichen Maßstabsebenen wird klassischerweise zwischen der lokalen, regionalen, nationalen und globalen Ebene unterschieden und es werden deren Interaktionen in der Geographie untersucht (Marston et al., 2009). Auch in der bildungsgeographischen Forschungspraxis erweist sich die Perspektive der Maßstabsebenen als geeignetes Instrument für die Analyse aktueller Transformationsprozesse in Bildungssystemen, die unter dem Einfluss von Wettbewerb und Internationalisierung stattfinden.

Mit wachsenden globalen Verflechtungen und einer damit einhergehenden time-space compression (Harvey, 1989) ist gleichzeitig eine zunehmende räumliche Entankerung ( $d i$ sembedding) alltagsweltlicher Bezüge (Werlen, 2001) zu beobachten. In der Analyse ergibt sich die Notwendigkeit einer entsprechend flexibleren Verknüpfung der geographischen Maßstabsebenen, wie sie etwa mit dem Konzept der „Gloka- 
lisierung“" (glocalization, Robertson, 1995) gefasst wird. Der Begriff bezeichnet zunächst die wechselseitige Interdependenz zwischen lokalen und globalen Strukturen und Prozessen, die durch unterschiedliche Dimensionen des Globalisierungsprozesses miteinander verbunden sind. Für die geographische Bildungsforschung bedeutet dies beispielsweise die Analyse von lokalen Bildungsprozessen in ihrer Interaktion mit bildungspolitischen oder ökonomischen Einflüssen auf der nationalen oder internationalen Ebene. Umgekehrt lassen sich auch internationale oder nationale Entwicklungen in ihren lokalen Auswirkungen oder institutionellen Umsetzungspraktiken erforschen.

Eine Tendenz ist der schleichende Rückzug vieler Nationalstaaten aus der flächendeckenden Bildungsversorgung sowie die Einführung von Markt- und Wettbewerbsmechanismen sowohl zwischen Hochschulen - insbesondere durch Evaluationen, Rankings und Studiengebühren - als auch zwischen Schulen, die zudem der Konkurrenz von privaten Bildungsunternehmen ausgesetzt sind. Diese Prozesse laufen in den einzelnen Ländern auf sehr unterschiedliche Weise ab: während beispielsweise für Großbritannien die Teilnahme an der global education industry (Tooley, 2001) einen wesentlichen Bestandteil der nationalen Wirtschafts- und Exportstrategie darstellt, verläuft die Ökonomisierung von Bildung in Deutschland bisher vergleichsweise zögerlich (Burs, 2013).

Mit Blick auf die handelnden Akteure des Bildungswesens hat die personenbezogene Mobilität in Studium und Wissenschaft inzwischen eine weltumspannende Dimension erreicht, welche nicht nur die Internationalität bestehender Hochschulstandorte erhöht, sondern darüber hinaus ein neues Phänomen in der globalen Hochschullandschaft hervorgebracht hat, das in der Fachliteratur als education hubs (Jöns, 2010) bezeichnet wird. Letztere sind vor allem auf der arabischen Halbinsel und im asiatischen Raum entstanden und verfolgen das Ziel, eine globale Studierendenschaft anzuziehen. Diese auch als transnational higher education spaces bezeichneten Konfigurationen werden als branch campuses verschiedener internationaler Hochschulen gemeinsam organisiert und stellen ein diversifiziertes hochwertiges Lehrangebot bereit. Anders als im Fall der distance education kommen Studierende und Lehrende in aller Regel für einen begrenzten Zeitraum zusammen, so dass der Hochschulstandort selbst lediglich die Infrastruktur bereitstellt (Knight, 2011).

In ähnlicher Form lassen sich auch im Schulbereich Formen der Internationalisierung erkennen, die als eine Verschiebung von Maßstabsebenen gelesen werden können. Unter dem Begriff der transnational spaces of education (Waters und Brooks, 2011) wird in der angelsächsischen Literatur u.a. die wachsende Verbreitung von internationalen Schulen untersucht. Ursprünglich wurden diese primär für Kinder von Expatriierten errichtet, besitzen aber heute in manchen Ländern den Status elitärer Bildungsinstitutionen für Familien der lokalen Bevölkerung, die ihren Kindern den $\mathrm{Zu}-$ gang zu einer globalen Elite ermöglichen wollen (Hayden, 2011). Auf diese Weise etabliert sich neben den historisch gewachsenen nationalen Bildungssystemen ein internationales Schulwesen, das der Reproduktion einer globalen Elite dient.

Auf der anderen Seite gibt es - neben der wachsenden internationalen Vernetzung, insbesondere von Hochschulen und vereinzelt auch von Schulen - eine Restrukturierung der nationalen Bildungssysteme, die in aller Regel mit einer Verlagerung der Steuerungsmacht von der nationalen auf die lokale Ebene verbunden ist. Beispielsweise hat sich in Ländern wie Schweden oder Finnland eine Kommunalisierung von Schulen und somit eine Steuerung auf Gemeindeebene durchgesetzt. Ebenso lassen sich in Deutschland erste Tendenzen der Reorganisation von Bildungsprozessen auf der lokalen Ebene erkennen. Mit diesen sogenannten lokalen oder regionalen „Bildungslandschaften“ oder „Bildungsnetzen" entwickeln sich neue Formen einer räumlich definierten „Bildungsgovernance“, die mit einem Rückzug der Länder aus der Bildungsverantwortung einhergeht.

\subsection{Grenzziehungen und Regionalisierungen}

Grenzziehungen und Regionalisierungen stellen zentrale Basiskonzepte geographischen Denkens und geographischer Forschung dar. Ausgehend von der grundlegenden Überlegung, dass Raumeinheiten und Grenzen immer sozial konstruiert sind und als Marker lediglich durch soziale Übereinkunft Bestand haben, beschäftigt sich die Geographie mit der Konstruktion und Dekonstruktion territorialer Grenzlinien sowie mit deren Auswirkungen auf Prozesse der sozialen und kulturellen Differenzbildung. In der politischen Geographie stehen dabei häufig nationalstaatliche Grenzen, deren Entstehung und die mit ihnen verbundenen sozialen Praktiken wie die Trennung des „Eigenen“ und des „Fremden“ im Vordergrund. In einem weiteren Sinne lassen sich auch andere Grenzen und Grenzziehungen als einfache Formen sozialer und politischer Ordnungen verstehen, durch die soziale Differenz markiert werden kann (Reuber, 2014; Secor, 2009; Sparke, 2009).

Bildungssysteme sind aufgrund ihrer Entstehungsgeschichte in besonderer Weise an nationalstaatliche Territorialgrenzen gebunden und spielen eine bedeutende Rolle in Prozessen der kulturellen Homogenisierung und nationalen Identitätsbildung. Denn die Einführung der Schulpflicht und der Ausbau nationaler Schulsysteme in der Frühzeit der Nationalstaaten hatte auch die Funktion, durch othering kulturelle Differenz gegenüber benachbarten Nationen in den Köpfen zu verankern und damit vorhandene politische Grenzlinien kulturell mit dem Blick auf nationale Identitäten zu markieren (Schiffauer, 2002). Infolge der deutschen Kulturhoheit der Länder, die auch das Bildungswesen betrifft, gibt es innerhalb des nationalen Bildungssystems erhebliche Unterschiede, deren Abbau und Homogenisierung sich oft als schwierig erweist. Die Persistenz länderspezifischer Schulbücher und Curricula, unterschiedlicher Schulformen und Schulzeiten sowie die bestehenden Schwierig- 
keiten bei der wechselseitigen Anerkennung von Abschlüssen oder Mobilitätsbarrieren für Lehrkräfte sind Ausdruck der historisch bedingten Heterogenität der deutschen Bildungslandschaft (vgl. Kramer und Nutz, 2010; Institut für Länderkunde et al., 2002). Mit dem Abbau der Grenzen innerhalb der Europäischen Union und dem Ziel der Schaffung eines europäischen Bildungsraums werden die nationalen Bildungssysteme vor neue Herausforderungen gestellt. Das bildungsgeographische Interesse richtet sich hierbei auf die innereuropäischen Grenzregionen als Kontaktzonen, da diese als Pionierräume supranationaler Bildungspolitiken und Bildungspraktiken angesehen werden können (Fichter-Wolf, 2010).

Jenseits der länderspezifischen Ausprägungen von Bildungspolitiken ist die Ausweisung von Schulbezirken ein Thema von hoher geopolitischer Relevanz, da hier Grenzziehungen vollzogen werden, die Auswirkungen auf soziales und räumliches Handeln haben können. Schulbezirke sind administrativ festgelegte räumliche Einheiten, welche die Zuordnung jedes einzelnen Wohnorts zu einem bestimmten Schulstandort festschreiben. Dieses klassische Instrument der Raumordnung kann sich sowohl auf Grundschulen als auch auf weiterführende Schulen beziehen. In Zeiten erhöhter Bildungssensibilität vermag die Reputation eines Schulstandorts das Wohnortwahlverhalten von jungen Eltern zu beeinflussen, während eine Veränderung der Schulbezirksgrenzen - wie das Beispiel der Berliner Bezirksreform gezeigt hat - soziale Proteste und Konflikte provoziert (Noreisch, 2007). Umgekehrt kann die Einführung des ,freien Elternwahlrechts" und damit die Aufhebung der Bindung an Schulbezirke im ländlichen Raum in Zeiten sinkender Schülerinnen- und Schülerzahlen auch zu einer Polarisierung und schließlich zur Aufgabe einzelner Schulstandorte führen (Frank, 2011). Schulpolitik wirkt sich somit unmittelbar auf die sozialräumliche Differenzierung von Städten und ländlichen Räumen aus, indem sie raumbezogenes Handeln beeinflusst und in bestehende sozialräumliche Gefüge eingreift.

\subsection{Räumliche Verankerung und Vernetzung}

Bildungseinrichtungen entstehen und entwickeln sich stets in einem spezifischen räumlichen Kontext, der durch historische, soziale, politische, kulturelle und ökonomische Rahmenbedingungen geprägt ist. Das Konzept der räumlichen Verankerung und Vernetzung bzw. geographischen Einbettung (embeddedness) wurde in verschiedenen wissenschaftlichen Kontexten aufgegriffen. Als Begriffspaar der ,Verankerung/Entankerung“ von traditionellen bzw. spätmodernen Gesellschaften hat Werlen mit Rückgriff auf Giddens die Einbettung traditioneller Lebensformen in einen spezifischen räumlichen Kontext beschrieben und diese den entankerten Lebensformen spätmoderner Gesellschaften gegenübergestellt (Werlen, 2000). In der Wirtschaftsgeographie wurde das Konzept der embeddedness mit Rückgriff auf Polanyi und Granovetter vor allem unter dem Aspekt der Einbettung und lokalen Vernetzung von Unternehmen und Institutionen in den Blick genommen (Hess, 2004).

Die Interaktion von formalen Bildungseinrichtungen mit ihren lokalen und regionalen Kontexten wurde in der deutschsprachigen Bildungsgeographie insbesondere für die Entwicklung einzelner Hochschulstandorte untersucht. Im Mittelpunkt standen dabei schon früh das Verhältnis von Universität und Stadt (Mayr, 1979) sowie die regionalökonomischen Auswirkungen von Hochschulen und Hochschulgründungen (Giese, 1987). Die komplexen sozialen und kulturellen Wechselwirkungen von Universitäten und ihrem unmittelbaren räumlichen Kontext rückten erst in Folge des cultural turn in den Mittelpunkt der Betrachtung. Beispielhaft dokumentiert der Wissenschaftsatlas der Universität Heidelberg (Meusburger und Schuch, 2011) die zahlreichen Dimensionen des Beziehungsgefüges zwischen Universität, Stadt und internationaler Vernetzung.

Auch im Bereich der Schulen ist das Konzept der räumlichen Verankerung und Vernetzung geeignet, um aktuelle Bildungsprozesse aus einer kritischen Perspektive zu beleuchten. Bildungsinstitutionen und schulische Bildungsangebote galten im Zusammenhang mit Fortschritt und Modernität lange Zeit als unumstritten positiv und wurden erst in der Folge von Postkolonialismus und Migration grundlegend in Frage gestellt. Die damit verbundenen Diskussionen um eine kulturelle Identitätsbildung deuten auf bestehende Konflikte hinsichtlich der Ausrichtung staatlich organisierter formaler Bildungsinstitutionen und informeller Bildungsinstitutionen - und dies gilt in besonderem Maße, wenn sich bestimmte soziale oder ethnisch-kulturelle Gruppen betroffen bzw. benachteiligt fühlen (Freytag, 2003a; Weber, 2014).

Ebenso wie Bildungsinstitutionen sind die Bildungsteilnehmerinnen und -teilnehmer im Sinne einer geographischen embeddedness verankert und vernetzt. Die sozialräumlichen Milieus, auch als Soziotope bezeichnet, beeinflussen das Aspirationsniveau der Lernenden sowie deren Einstellungen gegenüber dem Bildungswesen und den Bildungseinrichtungen. Ein Beispiel dafür ist die viel zitierte ,katholische Arbeitertochter vom Lande“, welche aufgrund ihrer räumlichen und sozialen Herkunft als ähnlich bildungsfern gilt, wie der „Migrantensohn“ im beginnenden 21. Jahrhundert (Geißler, 2005). Dabei handelt es sich um Prägungen, infolge derer die eigene Bildungsferne - ungeachtet einer selbst oder von den Kindern vollzogenen räumlichen Mobilität - mit einer gewissen Wahrscheinlichkeit auf die nachfolgenden Generationen übertragen wird. Umgekehrt zeigen Untersuchungen der asiatischen model minorities in den USA, dass ein Teil der Migrantinnen und Migranten mit besonders hohen Bildungsaspirationen überdurchschnittliche Erfolge im Bildungswesen zu erzielen vermag (Gamerith, 2005a).

Bildungsbeteiligung und Bildungserfolg lassen sich deshalb nicht als Ergebnis einer universellen Handlungslogik verstehen, sondern sie müssen innerhalb spezifischer Kontexte interpretiert werden. Aus kultur- und sozialgeographischer Perspektive geht es u.a. um Vertrauen bzw. Misstrau- 
en gegenüber Bildungseinrichtungen, Bildungsinhalten oder Bildungszertifikaten (Freytag, 2003a). Daraus ergeben sich durchaus unterschiedliche Rationalitäten im Hinblick auf die Bildungsaspirationen und -chancen. Letztlich wäre es zu einfach, einen formellen Bildungserwerb in normativer Weise uneingeschränkt positiv zu bewerten und den Bildungsteilnehmerinnen und -teilnehmern damit das Recht auf individuell gültige, kontextbezogene Bildungsrationalitäten absprechen zu wollen.

\subsection{Produktion und Repräsentation von Bildungsräumen}

Ein Teil des human- und vor allem kulturgeographischen Forschungsinteresses richtet sich seit einigen Jahren verstärkt auf räumliche Repräsentationen und die Produktion von Räumen auf diskursiver Ebene (Glasze und Mattissek, 2009). Dabei geht es einerseits um die Untersuchung raumbezogener Vorstellungen, die diskursiv mit bestimmten Eigenschaften und Bedeutungen in Verbindung gebracht werden. Andererseits wird thematisiert, wie bestimmte Sachverhalte, Strukturen und Prozesse diskursiv produziert und innerhalb konkreter raum-zeitlicher Kontexte verankert werden. Diese geographischen Forschungsansätze, deren methodische Ausrichtung eng an die benachbarten Sozial- und Kulturwissenschaften gebunden ist, besitzen ein bisher kaum genutztes Potential für bildungsgeographische Untersuchungen.

Ein Blick auf die bildungsbezogenen Diskurse im Bereich der deutschen Hochschulpolitik verdeutlicht diesen Zusammenhang. Während Hochschulstandortpolitik - aber auch Schulpolitik - im Zusammenhang der Bildungsexpansion der 1960er und 1970er Jahre noch unter dem Leitbild von Chancengleichheit und Gleichwertigkeit der Lebensbedingungen in allen Landesteilen stand, hat sich dieser Diskurs in den letzten beiden Jahrzehnten gewandelt. Heute ist die Repräsentation der deutschen Hochschullandschaft stärker von Wettbewerbsstrukturen und Eliteuniversitäten geprägt, die ihrerseits eher auf eine räumliche Ungleichverteilung hinwirken (Burs, 2013).

Auch auf internationaler Ebene ist derzeit eine strategisch angelegte, diskursive Produktion von Bildungsräumen zu beobachten, beispielsweise die Konstituierung eines europäischen Bildungsraumes im Zuge des Bologna-Prozesses. Die Existenz eines solchen Raumes wird zunächst in Diskursen und Strategiepapieren postuliert und auf diesem Wege im Bewusstsein der Menschen erzeugt, bevor durch begleitende Maßnahmen und Handlungen eine schrittweise Umsetzung dieser Vorstellungsbilder verfolgt wird.

Künftig werden kommunikative und diskursive Praktiken auch für Bildungspolitik und -marketing noch weiter an Bedeutung gewinnen. Gerade im Hinblick auf die Konstruktion von Bildungsräumen und lokalen Bildungslandschaften, die Überwindung von Grenzen und die diskursive Verräumlichung innerstädtischer Bildungsräume ergeben sich attraktive Forschungsfelder. Aus der Untersuchung dieser Repräsen- tationen und damit verbundener Praktiken könnte im Kontext des wachsenden Wettbewerbs um Forschende, Lehrende und Studierende eine kritische Bildungsgeographie erwachsen, die in Anknüpfung an die critical geography einen stärkeren gesellschaftspolitischen Anspruch vertritt.

\section{Fazit und Ausblick}

Im vorliegenden Beitrag wurden Überlegungen für eine theoretisch-konzeptionell fundierte Systematisierung bildungsgeographischer Forschungsarbeiten vorgestellt, welche den spezifischen Beitrag der Geographie zur Untersuchung aktueller Entwicklungen einer sich internationalisierenden und stark verändernden Bildungslandschaft postuliert.

Die Betonung und Sichtbarmachung sowie die Analyse räumlicher Disparitäten und regionaler Muster von Bildung ist ein Spezifikum bildungsgeographischer Untersuchungen und leistet zudem einen konstruktiven Beitrag zur räumlichen Bildungsplanung. Darüber hinaus erscheint es jedoch besonders wichtig, mit verschiedenen räumlichen Perspektiven auf die Komplexität aktueller Transformationsprozesse in ihrem gesellschaftspolitischen Kontext zu blicken, um auf diese Weise zu einem grundlegenderen Verständnis lokaler oder regionaler, vielleicht auch globaler Bildungslandschaften, Bildungsmilieus und Bildungsnetze zu gelangen.

Die in diesem Beitrag vorgeschlagenen sechs geographischen Konzepte orientieren sich an bestehenden bildungsgeographischen Forschungsarbeiten, gehen aber bewusst über die raumwissenschaftlichen Ansätze der klassischen Bildungsgeographie hinaus. Sie sind weder als abschließender Kanon noch als trennscharfe Forschungsfelder zu verstehen. Vielmehr besitzen sie eine heuristische Funktion, um die Anschlussfähigkeit der Bildungsgeographie an aktuelle humangeographische Entwicklungen zu fördern und gleichzeitig den spezifischen geographischen Beitrag zu einer interdisziplinären Bildungsforschung hervorzuheben.

Die zentrale gesellschaftliche und ökonomische Bedeutung, die Bildung heute zugeschrieben wird, hat weltweit zu umfassenden Restrukturierungsprozessen in nationalen Bildungssystemen geführt. Die damit verbundenen Tendenzen zur Deregulierung, Internationalisierung und Ökonomisierung bergen das Risiko neuer sozialräumlicher Fragmentierung und sozialer Exklusion infolge bildungspolitischer Eingriffe. In diesem Bereich besteht - gerade im deutschsprachigen Raum - noch erheblicher Bedarf an Forschungsarbeiten einer (gesellschafts-)kritischen Bildungsgeographie, die sich - unter Berücksichtigung der in diesem Artikel dargelegten Raumkonzepte - mit den sozialen und räumlichen Bedingungen und Auswirkungen dieser multiskalaren Prozesse auseinandersetzen.

Acknowledgement. Für die hilfreichen Kommentare und Anregungen, die wir im Verlauf der Ausarbeitung dieses Manuskripts erhalten haben, bedanken wir uns sehr herzlich. Unser besonderer 
Dank gilt Frau Katharina Hoffmann für die sorgfältige Durchsicht einer früheren Fassung des Manuskripts. Weiterhin möchten wir den drei anonymen Gutachter/-innen für ihre umsichtigen und konstruktiven Überarbeitungsvorschläge danken.

Edited by: B. Korf

Reviewed by: three anonymous referees

\section{Literatur}

Aurin, K.: Zur regionalen Differenzierung schulischen Begabungspotentials, Raumforschung und Raumordnung, 28, 202-211, 1970.

Autorengruppe Bildungsberichterstattung (Hrsg.): Bildung in Deutschland 2012. Ein indikatorengestützter Bericht mit einer Analyse zur kulturellen Bildung im Lebenslauf, http://www. bildungsbericht.de/daten2012/bb_2012.pdf (Zugriff am 25. Februar 2014), 2012.

Bahrenberg, G.: Zur Frage optimaler Standorte von Gesamthochschulen in Nordrhein-Westfalen. Eine Lösung mit Hilfe der linearen Programmierung, Erdkunde, 28, 101-114, 1974.

Barker, J., Alldred, P. und Watts, M.: Pupils or prisoners? Institutional geographies and internal exclusion in UK secondary schools, Area, 42, 378-386, 2010.

Burs, M.: Diskurs und Raum in der deutschen Hochschulentwicklung, Forum Politische Geographie, Band 8, Berlin und Heidelberg, Lit-Verlag, 2013.

Butler, T. und Hamnett, C.: The geography of education: introduction, Urban Stud., 44, 1161-1174, 2007.

Clifford, N. J., Holloway, S., Rice, S. P. und Valentine, G. (Hrsg.): Key concepts in geography, 2. Aufl., Los Angeles, Sage, 2009.

Cook, V. und Hemming, P.: Education spaces: the embodied dimensions and dynamics, Social and Cultural Geography, 12, 1-8, 2010.

Dörpinghaus, A. und Uphoff, I. K.: Grundbegriffe der Pädagogik, Darmstadt, WBG, 2012.

Fassmann, H., Kohlbacher, J. und Reeger, U.: Forgetting skills at borderline: foreign job seekers on the Viennese labour market, in: Skilled Migrations, Studi Emigrazione, Band 117, Herausgeber: Paganoni, T. und Todisco, E., Rom, Centro Studi Emigrazione, 78-89, 1995.

Fichter-Wolf, H.: Zur kommunikativen Konstruktion kulturräumlichen Wandels in Europa: Entwurf eines theoretischkonzeptionellen Analyseansatzes am Beispiel grenzüberschreitender Arrangements in der Hochschulbildung, Geogr. Helv., 65, 24-35, doi:10.5194/gh-65-24-2010, 2010.

Findlay, A. M.: From settlers to skilled transients: the changing structure of British international migration, Geoforum, 19, 401410, 1988

Flüchter, W.: Hochschulstandorte und Bildungsverhalten unter Aspekten der Raumordnung in Japan, Bochumer Geographische Arbeiten, Band 52, Paderborn, Schöningh, 1990.

Frank, K.: Schulentwicklungsplanung im demografischen Wandel. Am Beispiel der Kreise Dithmarschen und Steinburg, Dissertation, Kieler Arbeitspapiere, Band 52, Kiel, Geographisches Institut der Universität Kiel, 2011.

Frantz, K.: Washington Schools, Little White Man Schools und Indian Schools - Bildungsgeographische Fragestellungen dar- gestellt am Beispiel der Navajo Indianerreservation, Erde, 125 , 299-314, 1994.

Freytag, T.: Bildungswesen, Bildungsverhalten und kulturelle Identität: Ursachen für das unterdurchschnittliche Ausbildungsniveau der hispanischen Bevölkerung in New Mexico, Dissertation, Heidelberger Geographische Arbeiten, Band 118, Heidelberg, Selbstverlag des Geographischen Instituts der Universität Heidelberg, 2003a.

Freytag, T.: Mission schools, modernization, and mass education. Historical perspectives on a changing institutionalized education in New Mexico, in: Frontiers of geography. A selection from the wide range of geographical topics, Herausgeber: Nemes Nagy, J., Budapest, Eötvös Loránd Universität, 189-198, 2003b.

Friedrich, K. und Schultz, A. (Hrsg.): Brain drain oder brain circulation? Konsequenzen und Perspektiven der Ost-West-Migration, Forum IFL, Band 8, Leipzig, Institut für Länderkunde, 2008.

Gamerith, W.: Das Bildungssystem in den USA. Gute Schulen für alle?, Geographische Rundschau, 57, 38-45, 2005a.

Gamerith, W.: Ethnizität und Bildungsverhalten. Ein kritisches Plädoyer für eine „Neue“ Kulturgeographie, in: Bildung und Wissensgesellschaft, Heidelberger Jahrbücher, Band 49, Herausgeber: Kempter, K. und Meusburger, P., Heidelberg, Springer, 309332, $2005 b$.

Geipel, R.: Angewandte Geographie auf dem Feld der Bildungsplanung, in: Deutscher Geographentag in Bochum, 8.-11. Juni 1965. Tagungsbericht und wissenschaftliche Abhandlungen, Herausgeber: Monheim, F. und Beuermann, A., Wiesbaden, Steiner, 448-457, 1966.

Geipel, R.: Der Standort der Geographie des Bildungswesens innerhalb der Sozialgeographie, in: Zum Standort der Sozialgeographie. Wolfgang Hartke zum 60. Geburtstag, Münchner Studien zur Sozial- und Wirtschaftsgeographie, Band 4, Herausgeber: Ruppert, K. und Thiel, E., Kallmünz und Regensburg, Laßleben, 155-161, 1968.

Geipel, R.: Bildungsplanung und Raumordnung als Aufgaben moderner Geographie, Geographische Rundschau, 23, 15-26, 1969.

Geipel, R.: Die Universität als Gegenstand sozialgeographischer Forschung, Mitteilungen der Geographischen Gesellschaft in München, 56, 17-31, 1971.

Geipel, R.: Zur Entstehungsgeschichte des Forschungsansatzes „Geographie des Bildungsverhaltens“, Mitteilungen der Österreichischen Geographischen Gesellschaft, 118, 3-8, 1976.

Geißler, R.: Die Metamorphose der Arbeitertochter zum Migrantensohn. Zum Wandel der Chancenstruktur im Bildungssystem nach Schicht, Geschlecht, Ethnie und deren Verknüpfungen, in: Institutionalisierte Ungleichheiten. Wie das Bildungswesen Chancen blockiert, Herausgeber: Berger, P. A. und Kahlert, H., Weinheim und München, Juvenat, 71-100, 2005.

Giese, E.: Die Anziehungskraft wissenschaftlicher Hochschulen in der Bundesrepublik Deutschland für Studenten, Die Erde, 113, 115-132, 1982.

Giese, E. (Hrsg.): Aktuelle Beiträge zur Hochschulforschung, GieBener Geographische Schriften, Band 62, Gießen, Selbstverlag des Geographischen Instituts der Justus-Liebig-Universität GieBen, 1987.

Glasze, G. und Mattissek, A. (Hrsg.): Handbuch Diskurs und Raum. Theorien und Methoden für die Humangeographie sowie die sozial- und kulturwissenschaftliche Raumforschung, Bielefeld, Transcript, 2009. 
Güles, O., Wagener, T. und Wagner, R.: Bildung, Arbeit und Sozialraum. Zum besonderen Handlungsbedarf in benachteiligten Quartieren, Informationen zur Raumentwicklung, 2/3, 111-128, 2010.

Hanson Thiem, C.: Thinking through education: the geographies of contemporary educational restructuring, Prog. Hum. Geog., 33, 154-173, 2008.

Harvey, D.: The condition of postmodernity: an enquiry into the origins of cultural change, Oxford, Blackwell, 1989.

Hayden, M.: Transnational spaces of education. The growth of the international school sector, Globalisation, Societies and Education, 9, 211-224, 2011.

Hemming, P.: Educating for religious citizenship. Multiculturalism and national identity in an English multi-faith primary school, T. I. Brit. Geogr., 36, 441-454, 2011.

Hess, M.: "Spatial" relationships? Towards a reconceptualization of embeddedness, Prog. Hum. Geog., 28, 165-186, 2004.

Holloway, S. L. und Jöns, H.: Geographies of education and learning, T. I. Brit. Geogr., 37, 482-488, 2012.

Holloway, S., Hubbard, P., Jöns, H., und Pimlott-Wilson, H.: Geographies of education and the significance of children, youth and families, Prog. Hum. Geo., 34, 583-600, 2010.

Hoyler, M.: Räumliche und sozio-ökonomische Unterschiede des Analphabetismus zur Zeit der Industrialisierung. Das Beispiel der englischen Grafschaft Leicestershire 1754-1890, in: Beiträge zur Regionalen Bildungsforschung, Münchener Geographische Hefte, Band 72, Herausgeber: Geipel, R., Kallmünz und Regensburg, Laßleben, 97-130, 1995.

Hoyler, M.: Small town development and urban literacy. Comparative evidence from Leicestershire marriage registers 1754-1890, Hist. Soc. Res., 23, 202-230, 1998.

Institut für Länderkunde, Mayr, A. und Nutz, M. (Hrsg.): Nationalatlas Bundesrepublik Deutschland. Bildung und Kultur, Band 6, Heidelberg, Spektrum, 2002.

Jahnke, H.: Welcher Raum für welches Wissen? - Beobachtungen aus Berlin, Berichte zur deutschen Landeskunde, 78, 269-282, 2004.

Jahnke, H.: Der italienische Mezzogiorno auf dem Weg in die europäische Wissensgesellschaft. Eine Untersuchung der Erwerbssituation und der regionalen Mobilität junger Akademiker am Beispiel Siziliens, Dissertation, Berliner Geographische Arbeiten, Band 101, Berlin, Geographisches Institut der HumboldtUniversität zu Berlin, 2005.

Jahnke, H.: Bildung und Wissen, in: Schlüsselbegriffe der Kulturund Sozialgeographie, Herausgeber: Lossau, J., Freytag, T. und Lippuner, R., Stuttgart, Ulmer UTB, 153-166, 2014.

Johnston, R.: Spatial analysis, in: The dictionary of human geography, Herausgeber: Gregory, D., Johnston, R., Pratt, G., Watts, M. J. und Whatmore, S., 5. Aufl., Oxford, Wiley-Blackwell, p. 711, 2009.

Jöns, H.: Grenzüberschreitende Mobilität und Kooperation in den Wissenschaften. Deutschlandaufenthalte US-amerikanischer Humboldt-Forschungspreisträger aus einer erweiterten Akteursnetzwerkperspektive, Dissertation, Heidelberger Geographische Arbeiten, Band 116, Heidelberg, Selbstverlag des Geographischen Instituts der Universität Heidelberg, 2003.

Jöns, H.: Centre of calculation, in: The SAGE Handbook of Geographical Knowledge, Herausgeber: Agnew, J. A. und Livingstone, D. N., London, Sage, 156-168, 2010.
Klieme, E., Avenarius, H., Blum, W., Döbrich, P., Gruber, H., Prenzel, M., Reiss, K., Riquarts, K., Rost, J., Tenorth, H.-E. und Vollmer, H. J.: Zur Entwicklung nationaler Bildungsstandards. Expertise, Berlin, BMBF, http://www.bmbf.de/pub/zur_ entwicklung_nationaler_bildungsstandards.pdf (Zugriff am 25. Februar 2014), 2007.

Knight, J.: Education Hubs. A Fad, a Brand, an Innovation?, Journal of Studies in International Education, 15, 221-240, 2011.

Kraftl, P.: Geographies of alternative education. Diverse learning spaces for children and young people, Bristol, Policy Press, 2013.

Kramer, C.: Die Entwicklung des Standortnetzes von Grundschulen im ländlichen Raum. Vorarlberg und Baden-Württemberg im Vergleich, Dissertation, Heidelberger Geographische Arbeiten, Band 93, Heidelberg, Selbstverlag des Geographischen Instituts der Universität Heidelberg, 1993.

Kramer, C.: Jugendliche ohne allgemeinbildenden Schulabschluss. Regionale Disparitäten eines tabuisierten Problems, Europa Regional, 6, 15-24, 1998.

Kramer, C. und Nutz, M.: Bildung und Kultur, in: Deutschlandatlas. Unser Land in 200 thematischen Karten, Herausgeber: Hänsgen, D., Lentz, S. und Tzschaschel, S., Darmstadt, Primus, 85-104, 2010.

Marston, S., Woodward, K. und Jones III, J. P.: Scale. In: The dictionary of human geography, Herausgeber: Gregory, D., Johnston, R., Pratt, G., Watts, M. J. und Whatmore, S., 5. Aufl., Oxford, Wiley-Blackwell, 664-666, 2009.

Mayr, A.: Die Ruhr-Universität Bochum in geographischer Sicht. Stellung, Einzugsbereich und Standortproblematik einer neuen Hochschule, Berichte zur deutschen Landeskunde, 44, 221-244, 1970.

Mayr, A.: Universität und Stadt. Ein stadt-, wirtschafts- und sozialgeographischer Vergleich alter und neuer Hochschulstandorte in der Bundesrepublik Deutschland, Münstersche Geographische Arbeiten, Band 1, Paderborn, Schöningh, 1979.

Meusburger, P.: Landes-Schulentwicklungsplan von Vorarlberg, Bildungsplanung in Österreich, Band 3, Wien, Österreichischer Bundesverlag, 1974.

Meusburger, P.: Entwicklung, Stellung und Aufgaben einer Geographie des Bildungswesens. Eine Zwischenbilanz, Mitteilungen der Österreichischen Geographischen Gesellschaft, 118, 9-54, 1976.

Meusburger, P.: Beiträge zur Geographie des Bildungs- und Qualifikationswesens. Regionale und soziale Unterschiede des Ausbildungsniveaus der österreichischen Bevölkerung, Innsbrucker Geographische Studien, Band 7, Innsbruck, Selbstverlag des Institutes für Geographie der Universität Innsbruck, 1980.

Meusburger, P.: Die Heidelberger Professoren im Jahre 1984 mit besonderer Berücksichtigung ihrer regionalen und sozialen Herkunft, Beiträge zur Hochschulforschung, 1/2, 63-106, 1986.

Meusburger, P.: Bildungsgeographie. Wissen und Ausbildung in der räumlichen Dimension, Heidelberg, Spektrum, 1998.

Meusburger, P.: The spatial concentration of knowledge. Some theoretical considerations, Erdkunde, 54, 352-364, 2000.

Meusburger, P.: Sachwissen und symbolisches Wissen als Machtinstrument und Konfliktfeld. Zur Bedeutung von Worten, Bildern und Orten bei der Manipulation des Wissens, Geogr. Z., 93, 148 164, 2005. 
Meusburger, P.: Geography of Knowledge, in: Encyclopedia of Geography, Band 4, Herausgeber: Warf, B., Los Angeles, London, Sage, 1659-1663, 2010.

Meusburger, P. und Schuch, T. (Hrsg.): Wissenschaftsatlas der Universität Heidelberg. 625 Jahre Ruperto Carola, Knittlingen, Bibliotheca Palatina, 2011.

Meusburger, P. und Schmude, J. (Hrsg.): Bildungsgeographische Studien über Baden-Württemberg, Heidelberger Geographische Arbeiten, Band 88, Heidelberg, Selbstverlag des Geographischen Instituts der Universität Heidelberg, 1990.

Meusburger, P., Welker, M. und Wunder, E. (Hrsg.): Clashes of Knowledge. Orthodoxies and heterodoxies in science and religion, Knowledge and Space, Band 1, Dordrecht, Springer, 2008.

Monheim, H.: Die raumordnungspolitische Relevanz des Hochschulbaus. In: Informationen zur Raumentwicklung, 3/4, 201219, 1977.

Monheim, H.: Chancen für die weitere Entwicklung der neuen Hochschulen im ländlichen Raum, Informationen zur Raumentwicklung 4, 285-320, 1983.

Müller, S.: Bildungsstandorte und demographischer Wandel. Langfristig optimale Schulstandorte unter Berücksichtigung schwankender Schülerzahlen, Standort, 34, 6-10, 2010.

Noreisch, K.: Choice as Rule, Exception and Coincidence. Parents' Understandings of Catchment Areas in Berlin, Urban Stud., 44, 1307-1328, 2007.

Nutz, M.: Räumliche Mobilität der Studierenden und Struktur des Hochschulwesens in der Bundesrepublik Deutschland. Eine Analyse des Entscheidungsverhaltens bei der Studienortwahl und der Einzugsgebiete der Universitäten, Dissertation, Kölner Geographische Arbeiten, Band 54, Köln, Geographisches Institut der Universität zu Köln, 1991.

OECD (Hrsg.): Bildung auf einen Blick 2013. OECD-Indikatoren, Bielefeld, Bertelsmann, http://www.oecd-ilibrary.org/education/ bildung-auf-einen-blick-2013-oecd-indikatoren_eag-2013-de (Zugriff am 25. Februar 2014), 2013.

Pfaffenbach, C.: Wissen und Macht im Kontext von Remigration und Kommunalentwicklung. Konzeptionelle Überlegungen und empirische Befunde aus Syrien, Geogr. Z., 94, 160-175, 2006.

Picht, G.: Die deutsche Bildungskatastrophe, Olten, Wachter, 1964.

PISA-Konsortium Deutschland (Hrsg.): PISA 2003. Der zweite Vergleich der Länder in Deutschland - Was wissen und können Jugendliche?, Münster, Waxmann, 2005.

Pries, L.: Transnationalismus, Migration und Inkorporation. Herausforderungen an Raum- und Sozialwissenschaften, Geographische Revue, 5, 23-40, 2003.

Reuber, P.: Territorien und Grenzen, in: Schlüsselbegriffe der Kultur- und Sozialgeographie, Herausgeber: Lossau, J., Freytag, T. und Lippuner, R., Stuttgart, Ulmer UTB, 182-195, 2014.

Rhode, B.: Brain drain, brain gain, brain waste. Reflections on the emigration of highly educated and scientific personnel from Eastern Europe, in: The new geography of European migrations, Herausgeber: King, R., London, Belhaven Press, 228-245, 1993.

Robertson, R.: Glocalization. Time-Space and HomogeneityHeterogeneity, in: Global Modernities, Herausgeber: Featherstone, M., Lash, S. M. und Robertson, R., London, Sage, 25-44, 1995.

Rolfes, M.: Regionale Mobilität und akademischer Arbeitsmarkt. Hochschulabsolventen beim Übergang vom Bildungs- in das Beschäftigungssystem und ihre potentielle und realisierte Mobili- tät, Osnabrücker Studien zur Geographie, Band 17, Osnabrück, Rasch, 1996.

Schamp, E. W.: Universities as local knowledge centres in peripheral regions. The case of Göttingen, in: Regional and local economic policies and technology, Nederlandse Geografische Studies, Band 99, Herausgeber: Smidt, M. de und Wever, E., Amsterdam, Royal Netherlands Geographical Society, 115-122, 1989.

Schamp, E. und Zajontz, Y.: Wissensorganisation Universität und regionale Entwicklung im afrikanischen Kontext, am Beispiel der Universität Ngaoundere, Nordkamerun, Geogr. Z., 98, 133154, 2010.

Schiffauer, W.: Einleitung: Nationalstaat, Schule und politische Sozialisation, in: Staat - Schule - Ethnizität. Politische Sozialisation von Immigrantenkindern in vier europäischen Ländern, Herausgeber: Schiffauer, G., Baumann, G., Kastoryano, R. und Vertovec, S., Münster, Waxmann, 1-22, 2002.

Schmude, J.: Die Feminisierung des Lehrberufs an öffentlichen, allgemeinbildenden Schulen in Baden-Württemberg, Dissertation, Heidelberger Geographische Arbeiten, Band 87, Heidelberg, Selbstverlag des Geographischen Instituts der Universität Heidelberg, 1988.

Schultz, A.: Brain drain aus Ostdeutschland? Ausmaß, Bestimmungsgründe und Folgen selektiver Abwanderung, Forschungen zur Deutschen Landeskunde, Band 258, Leipzig, Deutsche Akademie für Landeskunde, 2009.

Secor, A.: Difference, in: The dictionary of human geography, Herausgeber: Gregory, D., Johnston, R., Pratt, G., Watts, M. J. und Whatmore, S., 5. Aufl., Oxford, Wiley-Blackwell, 159-160, 2009.

Sparke, M.: Border, in: The dictionary of human geography, Herausgeber: Gregory, D., Johnston, R., Pratt, G., Watts, M. J. und Whatmore, S., 5. Aufl., Oxford, Wiley-Blackwell, 52-53, 2009.

Stehr, N.: Arbeit, Eigentum und Wissen. Zur Theorie von Wissensgesellschaften, Frankfurt/M., Suhrkamp, 1994.

Stehr, N.: Knowledge as capacity for action. Paper presented at OECD Workshop "New Indicators for the Knowledge-Based Economy", http://www.publications.gc.ca/Collection/Statcan/ 88F0017M/88F0017MIE1996002.pdf (Zugriff am 25. Februar 2014), 1996.

Steingrube, W.: Die Entwicklung des Standortsystems der Grundschulen im Landkreis Rotenburg/W. seit 1950, Berichte zur deutschen Landeskunde, 58, 97-127, 1984.

Terpoorten, T.: GIS-gestützte kleinräumige Analyse amtlicher Schuldaten. Ein Ansatz für ein flächendeckendes Bildungsmonitoring am Beispiel des Ruhrgebiets, Standort, 29, 196-198, 2005.

Tooley, J.: The Global Education Industry. Lessons from Private Education in Developing Countries, London, Institute of Economic Affairs, 2001

Wagner, H.: Bildung und Raum: Entwicklungen und Strategien in einer geographischen Forschungsrichtung, Osnabrücker Studien zur Geographie, Band 13, Osnabrück, Universität, Fachgebiet Geographie, 1993.

Ward, K.: Inequality, spatial, in: The dictionary of human geography, Herausgeber: Gregory, D., Johnston, R., Pratt, G., Watts, M. J. und Whatmore, S., 5. Aufl., Oxford, Wiley-Blackwell, 380381, 2009. 
Waters, J. und Brooks, R.: International/transnational spaces of education, Globalisation, Societies and Education, 9, 155-160, 2011.

Weber, E.: Migrationshintergrund von Lehrern: Ressource oder Hindernis? Eine bildungsgeographische Studie, Dissertation, Fakultät für Umwelt und Natürliche Ressourcen, Albert-LudwigsUniversität Freiburg, 2014.

Wehrhahn, R. und Sandner Le Gall, V.: Bevölkerungsgeographie, Darmstadt, WBG, 2011.

Weichbrodt, M.: Ein Leben lang mobil? Langfristige Schüleraustauschprogramme und die spätere Mobilität der Teilnehmer als Element gesellschaftlicher Transnationalisierung, Dissertation, Wissenschaftliche Schriften der WWU Münster, Band 13, Institut für Geographie, Westfälische Wilhelms-Universität Münster, http://nbn-resolving.de/urn:nbn:de:hbz:6-24349645405, Zugriff am 25 Februar 2014.

Weichhart, P.: Multilokalität - Konzepte, Theoriebezüge und Forschungsfragen, Informationen zur Raumentwicklung, 1/2, 1-14, 2009.

Weick, C.: Räumliche Mobilität und Karriere. Eine individualstatistische Analyse der baden-württembergischen Universitätsprofessoren unter besonderer Berücksichtigung demographischer Strukturen, Dissertation, Heidelberger Geographische Arbeiten, Band 101, Heidelberg, Selbstverlag des Geographischen Instituts der Universität Heidelberg, 1995.
Weinert, F. E.: Vergleichende Leistungsmessung in Schulen - eine umstrittene Selbstverständlichkeit, in: Leistungsmessungen in Schulen, Herausgeber: Weinert, F. E., Weinheim, Basel, Beltz, 17-31, 2001.

Weishaupt, H.: Bildung und Region, in: Handbuch Bildungsforschung, Herausgeber: Tippelt, R. und Schmidt, B., Wiesbaden, Verlag für Sozialwissenschaften, 217-231, 2009a.

Weishaupt, H.: Demographie und regionale Schulentwicklung, Z. Pädagogik, 55, 56-72, 2009 b.

Wenzel, H.-J., Thomas, E. und Rolfes, M.: Studenten und Universitätsabsolventen im westlichen Niedersachsen beim Übertritt ins Beschäftigtensystem und ihre berufliche Flexibilität und räumliche Mobilität, Raumforschung und Raumordnung, 48, 130-141, 1990.

Werlen, B.: Sozialgeographie. Eine Einführung, Bern, Haupt UTB, 2000.

Werlen, B.: Entankerung, in: Lexikon der Geographie, Band 1, Herausgeber: Brunotte, E., Gebhardt, H., Meurer, M., Meusburger, P. und Nipper, J., Heidelberg, Spektrum, p. 307, 2001. 